\title{
Pathogenesis of anti-Yo positive paraneoplastic cerebellar degeneration
}

\author{
Laura Pepler and Carol Armstrong
}

\begin{abstract}
This manuscript was prepared for $\mathrm{BIOM} * 4500$ Research in Biomedical Sciences under the supervision of Prof. Carol Armstrong, Department of Biomedical Sciences, Ontario Veterinary College.
\end{abstract}

\begin{abstract}
Paraneoplastic cerebellar degeneration (PCD) is a neurological disorder affecting the cerebellum and is believed to be immune-mediated. Anti-Yo PCD is associated with gynaecological and breast cancer tumours and the presence of these tumours is believed to invoke an immune response. The immune response includes both anti-Yo antibodies and cytotoxic T-lymphocytes that are specific for the Cdr2 antigen expressed in Purkinje cells of the cerebellum. Although both have been implicated in the pathogenic manifestation of PCD neither have been shown to be directly involved. The detection of the anti-Yo antibody greatly aids in the diagnosis of PCD and helps in uncovering the underlying neoplasm before it becomes symptomatic. Treatment of PCD has been targeted at suppressing the immune response but has been unsuccessful so far. The lack of a suitable animal model makes it difficult to study the pathogenic process directly leaving many questions unanswered about PCD pathogenesis.
\end{abstract}

$\mathrm{P}$ araneoplastic cerebellar degeneration (PCD) is a rare neurological disorder causing cerebellar dysfunction that occurs as the result of a malignant tumour without the direct involvement of the tumour with the cerebellum. PCD is not caused by metastasis or any other associated complications of cancer such as metabolic dysfunction, nutritional factors, infections or side effects of cancer drugs. PCD is part of a larger group of disorders known as paraneoplastic neurological syndromes (PNS) that affect anywhere from $1 \%$ to $3 \%$ of cancer patients. ${ }^{1}$ PNS was first described in 1948 by Denny-Brown. In his paper he described the symptoms of two patients with sensory ataxia who were found to have bronchogenic carcinoma at autopsy with no metastasis to the nervous system. At post-mortem examination, both patients' dorsal root ganglia had very few cells left. He concluded, based on similar findings in animals with pantothenic acid deficiency, that their abnormal sensory symptoms could be the result of a metabolic disorder. ${ }^{2}$ It was not until 1965 that anti-neuronal antibodies were identified in the serum of patients who had cancer associated neurological degeneration. ${ }^{3}$ From this finding it was proposed that an immune reaction against the tumour could be responsible for the damage exhibited in the central nervous system (CNS). Since then, activated cytotoxic T-lymphocytes (CTL) and activated microglia have been identified in PCD cerebella further indicating that PCD pathogenesis is immune mediated. ${ }^{4}$ Studying PCD is important as it provides insight not only into autoimmune neurologic disease but may provide a rare example of tumour immunity in humans, in which the immune system mounts a response to the tumour causing the death of tumour cells. ${ }^{5}$

It is believed antigens expressed by tumour cells induce an immune reaction. Antibodies specific for the tumour antigen are produced and these antibodies cross react with antigens that are expressed on Purkinje cells in the cerebellum leading to extensive Purkinje cell loss and to a much lesser extent, granule cell loss leading to cerebellar dysfunction. ${ }^{4}$ PCD patients' neurological symptoms appear to develop rapidly and individuals often become ataxic. The ataxia is characterized by the loss of coordination of their muscles and extremities causing them to have difficulty walking and standing on their own. PCD is also characterized by difficulty swallowing and talking as well as involuntary extraocular eye movements. ${ }^{6}$

Each PCD case is associated with a particular type of antibody and each antibody type is closely associated with a tumour type. These antibodies include anti-Yo which is associated mainly with gynaecological and breast cancers, anti-Hu which is mostly found in patients who have smallcell lung cancer, anti-Ri which is associated with breast cancer and anti-Tr which is most often associated with Hodgkin's. ${ }^{7}$ These different antibodies do not affect all of the same cells in the same regions of the CNS therefore the different types can sometimes be distinguished clinically by the presentation of different neurological symptoms. This review will focus on anti-Yo type $\mathrm{PCD}$ as it is the most prevalent. $^{8}$ It is almost exclusively found in women, as it is most commonly associated with gynaecological and breast 
cancer tumours, although there have been rare cases when the anti-Yo antibody was present in male patients serum. These males were found to have a type of adenocarcinoma associated with the anti-Yo antibody. ${ }^{9}$ Although it has been widely accepted that the cerebellar degeneration seen in PCD is immune-mediated, the mechanism by which the Purkinje cells die is still in question. This review will focus on the current theories about the mechanisms behind PCD pathogenesis as well as the difficulties in understanding the role the immune system plays in this pathogenesis.

\section{Purkinje cell antigens associated with PCD}

Anti-Yo antibodies react with 3 different intracellular cerebellar degeneration related antigens $(\mathrm{Cdr})$ in Purkinje cells. Anti-Yo can react with a $62 \mathrm{kDa}$ species known as Cdr2, a $52 \mathrm{kDa}$ species known as Cdr3 or a $34 \mathrm{kDa}$ species known as Cdr1. ${ }^{10}$ Structurally the Cdr2 antigen contains leucine residues on different helices that connect to form a zipper-like structure which stabilizes the protein. ${ }^{10}$ This is known as the leucine zipper motif and is common in DNAbinding proteins that affect gene transcription, however it has been shown the Cdr2 antigen associates not with DNA but with free ribosomes in the cytoplasm. ${ }^{11}$ The role Cdr2 may play in Purkinje cell death is unclear.

It is believed that anti-Yo antibody may not be the pathogenic culprit in PCD because it targets intracellular antigens and not membrane bound antigens. Results of animal studies on guinea pigs have shown that intraventricular injection of anti-Yo antibodies of the immunoglobulin class G1 accumulate in the Purkinje cell cytoplasm leading most to believe that these antibodies are capable of entering cells and affecting cellular function. ${ }^{12}$ The Cdr2 antigen is almost always expressed in ovarian and breast cancer tumours of those who develop PCD while the Cdr1 and Cdr3 antigens are expressed less frequently. ${ }^{13}$ The $\mathrm{Cdr} 2$ antigen always seems to be expressed in the tumours of those that develop PCD so it is believed the expression of this antigen is a critical step in the pathogenesis of PCD and the induction of synthesizing anti-Yo antibody, although is not a determinant that the expression of this antigen will lead to PCD.

A study looking at the expression pattern of the cdr2 gene found that mRNA for the cdr2 gene is found in virtually all cells throughout the human body, while the protein is expressed mainly in Purkinje cells of the cerebellum and to a lesser extent in neurons throughout the $\mathrm{CNS} .{ }^{14} \mathrm{Cdr} 2$ was also expressed in the testis which may explain the rare cases of the presence of anti-Yo in males. ${ }^{14}$ This restricted expression is likely due to a post-transcriptional modification within these sites. Both of these sites (CNS and testis) are considered to be immune-privileged sites, meaning the immune system and its factors should not have access. The cerebellum is supposed to be protected by the blood brain barrier (BBB) and therefore should be safe from circulating antibodies and other mediators of the immune system. However, this is not the case in PCD. There seems to be a breakdown of the BBB allowing immune mediators access to a normally restricted site. The Cdr2 antigen in Purkinje cells has been strongly implicated to play a role in Purkinje cell death but there has been no direct pathway determined for Purkinje neuron apoptosis. ${ }^{15}$ The function of $\mathrm{Cdr} 2$ has been under investigation for numerous years and it is believed through interactions with anti-Yo in the cytoplasm of Purkinje cells it could lead to Purkinje cell death. ${ }^{16}$

\section{Pathogenesis of PCD: Current Theories}

It is often the case that when proteins such as Cdr2 are restricted in their expression to immune-privileged sites, that the immune system does not have access to the antigens. When the Cdr2 antigen is expressed outside of an immuneprivileged site, such as when it is expressed in tumour cells, the body's immune surveillance mechanisms recognize the $\mathrm{Cdr} 2$ antigen on tumour cells as foreign. These antigens are highly immunogenic because they have been restricted in their expression, and are capable of invoking an immune response. It is important to identify possible mechanisms of PCD because an understanding of these mechanisms provides possible targets for treatment.

\section{Auto-antibody response}

Originally it was believed that PCD could only result from an auto-antibody response. The Cdr2 antigen in tumour cells would be recognized as foreign molecules and induce an immune response that would recruit B-cells to produce autoantibodies specific for this $\mathrm{Cdr} 2$ antigen. These antibodies would circulate in the periphery and cross the BBB into the cerebellum where they would accumulate in the Purkinje cells of the cerebellum and play a role in interfering with cellular function and possibly lead to Purkinje cell death by antibody toxicity. ${ }^{4}$ One antibody-mediated pathway leading to Purkinje cell death was proposed by Okano et al. ${ }^{16}$ who found the leucine zipper motif on Cdr2 directly interacted with another leucine zipper motif on a nuclear transcription factor known as c-Myc. The two proteins were shown to colocalize in the cytoplasm and to directly interact with each other by co-precipitation in vitro. Okano et al. ${ }^{16}$ also showed Cdr2 did not interact with other leucine zipper motifs on a series of other proteins demonstrating Cdr2 has specificity for c-Myc. Through a series of in-vitro experiments using rat cerebellum, Okano et al. ${ }^{16}$ were also able to demonstrate that the presence of $\mathrm{Cdr} 2$ in Purkinje cells causes c-Myc to redistribute from the nucleus to the cytoplasm. They were able to show this by expressing Cdr2 under a cytomegalovirus promoter in a neuroblastoma cell line. The cells that were not engineered to express Cdr2 showed c-Myc distribution limited to the nucleus and cells that were expressing Cdr2 showed c-Myc redistributed to the 
cytoplasm allowing for interaction. It is believed normal $\mathrm{Cdr} 2$ function regulates c-Myc entry into the nucleus and thus regulates the level of c-Myc associated signalling activities. To show the effects PCD may have, Okano et al. ${ }^{16}$ introduced PCD antiserum containing $\mathrm{Cdr} 2$ specific antibodies and this appeared to inhibit the interaction between $\mathrm{Cdr} 2$ and c-Myc as they were unable to coprecipitate as they had before. It is possible the binding of the anti-Yo antibodies to Cdr2 inhibits its interaction with c-Myc causing c-Myc entry into the nucleus to go unregulated, resulting in an excess of c-Myc in the nucleus. As a result of this excess, cell cycle signalling would be disrupted and cMyc may contribute to inducing apoptosis. c-Myc is also present in tumour cells that express Cdr2 where it has been shown to play a role in apoptosis. ${ }^{16}$ It would be of value to know if the relationship between c-Myc and Cdr2 in Purkinje neurons is similar to a relationship they have in tumour cells. There have also been studies looking at c-myc expression patterns in the developing cerebellum as well as the adult cerebellum which have shown higher c-myc expression in developing Purkinje neurons and lower levels in adult neurons. ${ }^{17}$ The low expression of c-myc in adult Purkinje neurons was characterized by groups of neurons expressing high amounts of c-myc while most others minimally expressed c-myc or did not express it at all. This observation suggests that Purkinje neurons may die in patterns or perhaps that expressing abnormally high amounts of c-myc in adult Purkinje neurons predisposes someone to developing PCD.

In a more recent study a similar mechanism was proposed for Purkinje cell death. Sakai et al. ${ }^{18}$ showed Cdr2 interacts with a mortality factor-like protein that has significant homology with MRG15, an important regulating factor in the cell cycle. This study suggests the Cdr2 antigen acts on MRG15 to decrease its displacement of E2F from Rb, two important factors in the apoptotic pathway: a decrease in MRG15 protects neurons from entering the apoptotic pathway. It is suggested the presence of anti-Yo antibodies interrupts the interaction between Cdr2 and MRG15 causing MRG15 to displace E2F from $\mathrm{Rb}$ and the presence of E2F alone could cause neuronal apoptosis. However, if $\mathrm{Cdr} 2$ does play a neural protective role as Sakai et al. ${ }^{18}$ suggest, then one would think Cdr2 would be expressed in all neurons and not limited to high expression in only Purkinje neurons.

Neither of these studies have shown that anti-Yo alone is directly causing Purkinje cell death and many believe that these antibodies do not play a direct role in the death of Purkinje cells for a number of reasons. First, animal studies have failed to replicate neurological symptoms in rats when antibodies are passively and actively transferred. ${ }^{19}$ Second, treatments aimed at decreasing anti-Yo antibody in the circulation have not been effective in alleviating the neurological symptoms and third, in about $2 \%$ of cases, patients with cancer who are also anti-Yo positive do not go on to develop PCD symptoms. ${ }^{7}$ These factors all suggest that there are other factors mediating the pathogenesis of PCD.

\section{Cytotoxic T-lymphocytes}

As is often the case in immunological based diseases, both the humoral and cellular branches of the immune system work together. In more recent years studies have focused more on the role cytotoxic T-lymphocytes (CTLs) may play in PCD pathogenesis. CTLs have been implicated because in many PCD patients Cdr2-specific CTLs have been detected in their blood as well as in CNS tissue in post-mortem studies. ${ }^{20} \mathrm{~A}$ study looking at CTL levels in PCD patients found that during the acute stages of the disease twenty to forty percent of cells found in the cerebrospinal fluid (CSF) were activated CTLs. ${ }^{21}$ This suggests the CTLs are breaching the BBB. Since the brain is an immune privileged site, no CTL should have access to CSF. A study found that activated non-neural specific T-cells had an effect on the integrity of the BBB and found that it was made more permeable to circulating antibodies, and perhaps more permeable to other immune system cells and factors as well. ${ }^{22} \mathrm{~A}$ breach in the BBB could allow the CTLs to gain access to the CSF and the release of cytokines that could cause an immune reaction in the CNS. It has been shown in vitro that the expression of MHC class 1 on Purkinje neurons, which is important in target recognition, can be pathologically induced. ${ }^{23}$ The expression of MHC class 1 that has been seen on Purkinje neurons could be the result of a breach in the BBB and result in the Purkinje neurons becoming targets for the immune response leading to Purkinje cell death.

In contrast, other findings have shown no inflammatory infiltrates around the cerebellum. ${ }^{8}$ This could be due to the stage of the disease which would correlate with the amount of inflammatory infiltrates found in the cerebellum. A patient who has progressed into the chronic stage may no longer be experiencing Purkinje cell death as the pathogenesis has ended and therefore would have no inflammatory infiltrates. Through numerous case studies it has been shown the onset of PCD symptoms is quick, followed by symptoms stabilizing where they neither get better or worse, perhaps indicating that the pathogenesis is no longer taking place. ${ }^{8}$ The reason PCD symptoms stabilize is unclear. Stabilization of symptoms could mean that not all Purkinje cells are dying and there are still functional cells or that all Purkinje cells have already died.

For a CTL to become activated and have cytotoxic capabilities it is necessary for some event to occur where an antigen-presenting cell takes up the $\mathrm{Cdr} 2$ antigen and then presents the antigen to a CTL. The CTL needs to recognize its target cell, in this case the Purkinje neuron, through an MHC class 1 molecule. What is unclear about such a mechanism is what event causes the antigen-presenting cell to take up the antigen and present it in the first place and where this event takes place. It has been proposed that when tumour cells containing the $\mathrm{Cdr} 2$ antigen migrate to the lymph nodes they are capable of peripherally activating CTLs through the antigen-presenting cells known as 
dendritic cells. ${ }^{20}$ These activated CTLs would now be specific for the Cdr2 antigen in Purkinje cells. It is interesting to note that in one study, Rojas et al. ${ }^{24}$ found that at the time of diagnosis of PCD patients with breast cancer, the tumours had already metastasized to the lymph nodes suggesting that it is the lymph nodes where the immune response may be triggered. Activated CTLs will then interact with a surface antigen MHC I that was identified as being expressed on neurons when it was previously thought not to be since the CNS is an immune-privileged site. ${ }^{23}$ The CTLs would then interact with the Purkinje cells and contribute to their death. It has also been suggested by Okano et al. ${ }^{16}$ that even a rare event such as an antibody mediated apoptotic event of a Purkinje neuron could initiate CTL activation. Giometto et al. ${ }^{4}$ identified increased activated microglia cells in the cerebellum of PCD patients through immunohistochemistry. Activated microglia have phagocytic abilities to take up apoptotic debris, including the Cdr2 antigen, and present the antigen to activate CTLs. An event such as this that takes place in a localized area in the cerebellum would help explain why PCD pathogenesis is greatly restricted to the Purkinje cells in the cerebellum and does not seem to affect other neurons which may express lower levels of $\mathrm{Cdr} 2$ throughout the CNS. However like antibodies, CTLs have not yet been shown to directly contribute to Purkinje cell death.

\section{Expression of CD59 on Purkinje neurons}

CD59 is an enzyme on the surface of cells which inhibits complement activation, which is a series of enzymatic reactions that lead to the formation of the membrane attack complex (MAC). The MAC, if allowed to assemble on the surface of cells will lead to cell death. In a recent study looking at the heterogeneous expression of CD59 on Purkinje cells in post-mortem tissue, CD59 was absent on the surviving Purkinje neurons in those afflicted with PCD. ${ }^{25}$ This was in comparison to Purkinje neurons that did express CD59 in a patient suffering multiple sclerosis, as well as those with normal cerebellar function, although not all Purkinje neurons will express CD59. Purkinje cells without CD59 may be left more vulnerable to cell death via the MAC. The expression of CD59 can be influenced by cytokines, like interferon-gamma which has been shown to decrease CD59 expression. ${ }^{26}$ It is not unreasonable to propose that the activated CTLs in the periphery may play a role in the release of cytokines that cross the BBB and interrupt the expression of CD59. Another observation made by Storstein et al. ${ }^{25}$ was the finding of MAC components in the cerebellum of patients with PCD suggesting the MAC complex had assembled and perhaps was the cause of Purkinje cell death. However, because these were postmortem findings it is difficult to determine whether the absence of CD59 is the cause of cell death or a result of the disease process that had already taken place or even if CD59 was present on the Purkinje cells before they died. Storstein et al. ${ }^{25}$ also note that the expression of complement regulators like CD59 in the cerebellum is sparse to begin with, because of it being considered immune privileged, and that under normal circumstances there should be no need for complement activation. It also should be mentioned that this study only looked at two PCD patients who as a result of their PCD had few Purkinje cells left, which could contribute to the absent CD59 on the remaining Purkinje cells. These two factors could be reasons for such little expression of CD59 to begin with. It would be of value to know if any heterogeneity exists in CD59 levels among those that test positive for anti-Yo and develop PCD as well as the ones that test positive for anti-Yo and do not develop PCD.

\section{Diagnosis}

The neurological symptoms associated with PCD often develop rapidly, with onset in only one day in some individuals and up to sixteen weeks in others. ${ }^{8}$ This shows the variability of the disease and suggests the pathogenic process may be prolonged in some and perhaps may start before neurological symptoms appear. The rapid onset of symptoms associated with PCD can also lead to a wrong diagnosis of a stroke that has affected cerebellar function. PCD is characterized by ataxia of the trunk and limbs, involuntary eye movements (nystagamus), slurring of speech and difficulty swallowing (dysphagia). ${ }^{6,7,8}$ The acute onset of these symptoms is then followed by a period of stabilization; however by this point most PCD patients are left with an inability to walk or stand on their own, and are unable to eat or drink and can no longer live independently. In one study the majority of PCD patients reached their greatest disability in just 4 months. ${ }^{24}$ In anti-Yo associated PCD, the neurological symptoms appear before the associated gynaecological tumour is detected $65 \%$ of the time. ${ }^{8}$ This delay between the onset of neurological symptoms and the detection of the underlying neoplasm is on average 3 months but can be as long as 5 years. $^{24}$ This delay between neurological symptoms and diagnosis of cancer is an important observation for a few reasons. It is an advantage to recognize and diagnose $\mathrm{PCD}$ quickly so the search for the underlying neoplasm can be found and the appropriate cancer treatment can begin. It is also important to recognize PCD symptoms early in an effort to prevent further irreversible cerebellar degeneration from occurring.

The evidence suggesting the tumour is not detectable through a symptomatic way at the onset of neurological symptoms has sparked interest in the idea that patients with PCD may be exhibiting a form of tumour immunity. It seems that when PCD patients are diagnosed, $90 \%$ are found to have a limited stage tumour (cancer found in only one organ or in a lymph node close by) whereas a diagnosis of cancer, with or without PCD, presents with a limited stage tumour only $50 \%$ of the time. ${ }^{20}$ There is some controversy over this 
as Rojas et al., ${ }^{24}$ found no discrepancies in the size or stage of the tumours. It is reasonable to think that those who are affected with PCD will have their cancer diagnosed earlier because of the presence of neurological symptoms indicating an underlying tumour whereas those who do not have PCD would most likely wait for a symptomatic sign of the tumour. This would explain the discrepancy, without implicating tumour immunity mechanisms for the difference in stages at presentation. MRI and CT scans are relatively useless in uncovering the aetiology of neurological symptoms. ${ }^{1}$ Scans of the brain usually appear normal and generally do not indicate cerebellar atrophy even though there is extensive loss of Purkinje cells.

Once anti-Yo antibody is detected in a patient's serum or CSF, it is a good indicator that the patient has PCD. Only about $2 \%$ of individuals that test positive for anti-Yo and have an underlying neoplasm do not develop neurological symptoms. ${ }^{27}$ Whether neurological symptoms appear or not, if anti-Yo is detected by immunohistochemistry, Western Blot is used to confirm antibody specificity and is required for a diagnosis of PCD. ${ }^{7}$ Since anti-Yo is essentially being used to identify the type of associated cancer, the detection of antibodies is important in the diagnostic process. The reliance upon the presence of anti-Yo to diagnose PCD is possible because anti-Yo is almost always associated with gynaecological and breast cancers, and because anti-Yo is almost never present in patients without cancer. ${ }^{28}$ One study gives a figure of about $2 \%$ of patients will be positive for anti-Yo with no detectable tumour, ${ }^{28}$ while others say it never happens. ${ }^{7}$ This discrepancy could be explained by how long after the initial detection of anti-Yo the health of the patients was followed up on by their physicians, as it has been shown that in extreme cases in can take up to 5 years for the cancer to become evident. ${ }^{24}$ The discrepancy could also be due a case of tumour immunity, where the immune reaction attacks the tumour cells and gets rid of the tumour. Either way the presence of anti-Yo has been shown to be confirmatory of a PCD diagnosis. In addition, the CSF of PCD patients should also indicate increased levels of CTLs, and CA125 (an ovarian tumour marker) which are usually increased in a PCD patients serum. ${ }^{29}$

Anti-Yo antibody is not detectable in healthy individuals and is almost always associated with gynaecological or breast cancer tumours. ${ }^{28}$ This high degree of specificity of the anti-Yo antibody greatly aids in the diagnosis of PCD and helps in searching for the associated tumour. However, because anti-Yo has a high specificity for being associated with gynaecological or breast cancer, the presence of anti-Yo is enough to begin searching for an underlying neoplasm even though one might not be readily evident through the use of CT or MRI scans of the abdominal and pelvic areas. When patients do test positive for anti-Yo and an MRI or CT scan does not uncover a tumour a fluorodeoxyglucose positron emission tomography (PET) scan should be used. ${ }^{1}$ This scan is capable of detecting small tumours by measuring metabolically active tissue. Its use in PCD patients prevented unnecessary surgery in $20 \%$ of patients who were believed to have a tumour but CT and MRI scans failed to detect it, and therefore should be implemented as a standard procedure despite its cost. ${ }^{30}$ However if a PET scan fails then surgical means may be required to find the tumour since the presence of anti-Yo signifies that there is a tumour present. ${ }^{29}$ In one case study, a woman who tested positive for anti-Yo antibody and was exhibiting typical PCD neurological symptoms such as ataxia was undergoing MRI and CT scans repeatedly at follow ups but nothing appeared to be abnormal. ${ }^{31}$ There was one enlarged lymph node that was linked to the lymphatic drainage of the pelvic area, and tests to measure the ovarian tumour marker CA125 indicated that it was elevated. This was considered enough evidence for this woman to receive an ovariectomy with out any confirmatory evidence of a tumour. Upon removal of the ovary and associated structures, there appeared to be no abnormalities macroscopically but under further microscopic examination they found an advanced staged tumour. Thus it is important to find and identify the tumour as quickly as possible because by treating the tumour it offers the greatest chance of treating and preventing further neurological damage.

Because of the many different PNS and different antineuronal antibodies that are associated with PNS, there has been a lack of criteria to go about diagnosing PNS and more specifically anti-Yo PCD. A panel of neurologists discussed what this criterion should be to make a diagnosis of classical PCD. ${ }^{28}$ They include the development of severe neurological symptoms in less than 12 weeks with symptoms that interfere significantly with lifestyle and no cerebellar atrophy (measured through CT scans of brain) within early stages of onset. Although this is helpful in identifying PCD it is important to remember the variability of the disease. For example, there have been reports of those diagnosed with PCD who do exhibit cerebellar atrophy. ${ }^{32}$ By sticking to such rigorist criteria, it could lead to a missed diagnosis of a patient who might not quite fit the description of classical PCD.

\section{Treatment}

The evidence thus far suggests the development of PCD is immune mediated, so treatments have primarily been aimed at suppressing the immune response. Since both antineuronal antibodies and CTLs have been implicated to play a role in the pathogenesis of PCD, they have been the main targets of treatment. These treatments include plasma exchange, immunosuppression using corticosteroids, decreasing $\mathrm{T}$-cells through drugs that affect gene transcription within these cells, immunoglobulin injections and removal of the tumour either through chemotherapy or surgery. ${ }^{1}$ These treatments on their own and in combination have been widely unsuccessful. 
In one study that followed 34 patients with PCD, the outcome of one of the above treatment methods was compared to different combination therapies. ${ }^{24}$ Not one of the 34 patients showed any improvement - and the average survival for patients with anti-Yo positive PCD was 22 months, which is less then some other PCDs that are characterized by different anti-neuronal antibodies specific for different tumours. The only shorter survival rate was PCD associated with small cell lung cancer with the anti-Hu antibody. The cause of death in 9 of the patients was the neurological damage caused by PCD. This study ultimately shows the prognosis for anti-Yo positive PCD is quite poor.

Efforts to decrease activated CTLs in PCD patients did not show any promise as an effective treatment either. PCD patients were treated with an inhibitor of activated CTLs known as tacrolimus. ${ }^{21}$ This did dramatically decrease the number of activated CTLs from $30 \%$ of all cells in the CSF before treatment to less than $1 \%$ after treatment. However, tacrolimus did not have an effect on stopping peripheral CTLs from being activated again. Once treatment was stopped the number of activated CTLs rose again. This treatment also did not have an effect on the neurological symptoms as the patients that were treated had already suffered irreversible neurological damage. It is unclear if this course of treatment was able to prevent any further neurological damage because no controls were used in the study.

There have been isolated cases where a PCD patient's neurological condition does improve through treatment efforts. A woman diagnosed with ovarian cancer and experiencing PCD symptoms including ataxia that left her bedridden, began a course of treatment within 1 month of diagnosis that included intravenous methylprednisone, which is a potent immunosuppressant, and intravenous immunoglobulin. ${ }^{33}$ The immunoglobulin acts as a block between CTLs and antigen interaction, effectively decreasing the CTLs in the circulation and CSF. After just 2 days after treatment began, the woman showed remarkable improvement and was able to stand with assistance, something she was unable to do before. This improvement was sustained at a 5 month follow-up and even though she was still suffering from ataxia it was less severe then when she originally presented with the condition.

In contrast, a PCD patient in the same study started on intravenous immunoglobulin 2 weeks after diagnosis, did not show any improvement and even deteriorated. However this patient's tumour had been present for numerous years which could affect how well the treatment works. In the Rojas et al. ${ }^{24}$ study, 23 of the 34 patients were given intravenous immunoglobulin and all 23 showed no improvement. The outcomes of these studies show the unpredictable nature of treating PCD, and that cases showing improvement are quite isolated.
Another study looking at treatment effects on PCD patients showed 2 out of 4 patients who underwent immunosuppression experienced stabilization of their symptoms, while 2 out 4 patients who received no treatment also observed stabilization of the disease. ${ }^{34}$ It is unclear in some of these studies if the improvement seen is really due to treatment or just due to the observation that PCD symptoms, after a rapid onset, do stabilize. It is logical to think that starting treatment soon after the onset of symptoms would be most beneficial in hopes of stopping the progression of the disease. Widdess-Walsh et al. ${ }^{33}$ suggests that early treatment (less than 1 month after onset of symptoms) with high doses of intravenous immunoglobulin as well as treatment of the underlying tumour is the best chance of halting the progression and that treatment 3 months after diagnosis would yield a poor prognosis.

It is clear that there are many factors which can influence the outcome of treatment. Some of the factors which have been studied are the effects of age, sex, amount of time elapsed before treatment, type of tumour, treatment of tumour, and which antibodies are present. In a study done by Candler et al., ${ }^{35}$ the only factor associated with any marked stabilization of a patient's condition was the treatment of the underlying tumour. This is evident in a study that followed 137 PCD patients, 19 of whom were confirmed to be anti-Yo positive, in which the only individual that did improve received treatment for the underlying tumour (however the improvement was not significant). ${ }^{34}$ On the other hand, there are also descriptions of patients who were undergoing antineoplastic treatment who began to develop neurological symptoms indicative of PCD. ${ }^{24}$ This discrepancy could be due to the different types of tumours being treated, and the difficulties in treating tumours that have already metastasised. Implementing immunosuppression as a treatment for PCD in a patient who has cancer seems counterintuitive, and theoretically would aggravate the cancer even more and allow the tumour to grow. However in one study where immunosuppression was the course of treatment there was no recurrence of the tumour but this was an isolated case and it is unclear if this is the exception or the rule. ${ }^{21}$ It could be the reason that treatment that is targeted at immunosuppresion has been so unsuccessful. Others have proposed that the mechanisms by which immunotherapies act is much too slow to treat the rapid immune reaction observed in those with PCD. ${ }^{24}$

Perhaps there is more to the pathogenesis of PCD then just the immune system. Whatever the reason is, treatment targeting the immune reaction has been unsuccessful. The next step in treatment is looking at the possibility of implementing aggressive immunosuppression by eliminating the bone marrow and using haematopoietic stem cell transplantation to replace it. ${ }^{24}$ There are currently no protocols for this type of treatment to be used in PCD 
patients but it may be the next step in treatment of PCD.

\section{Limitations in Studying the Pathogenesis OF PCD}

Studying the pathogenesis of PCD has been complicated by a number of factors, the main one being the lack of a suitable animal model for PCD. Because there currently is no animal model for PCD it has made it difficult to directly study the immune mechanisms that are believed to result in Purkinje cell death. An in vivo study was able to induce antibodies and CTLs specific for the Cdr2 antigen by immunizing mice by a gene gun loaded with DNA that encodes cdr2. ${ }^{36}$ They found that immunization resulted in high levels of $\mathrm{Cdr} 2$ antigen in dendritic cells in the lymph nodes throughout the mice, indicating this may be the site of antigen induced activated CTLs. The mice were observed for the development of neurological symptoms for a year after the first immunization event and then sacrificed and sections of their cerebella were analysed. There did not appear to be any Purkinje cell loss or immune system cells present in the cerebellum, and there were no observed neurological effects of the induced immune response. ${ }^{18}$

More recently another attempt to replicate PCD was performed, this time using yeast as the delivery method of the $\mathrm{Cdr} 2$ antigen. ${ }^{19}$ Yeast possesses immunostimulatory mechanisms and because it uses live cells to express the $\mathrm{Cdr} 2$ antigen it more closely resembles the development of PCD in humans then previous attempts did. Sakai et al. ${ }^{19}$ used recombinant yeast that contained the $\mathrm{Cdr} 2$ antigen which induced both antibodies and CTLs specific for the $\mathrm{Cdr} 2$ antigen. However this latest attempt again did not result in any clinical signs of PCD or loss of Purkinje cells. These findings suggest that there are other factors mediating PCD pathogenesis and that both anti-neuronal antibodies and activated CTLs are not enough to cause the pathological manifestation of PCD. The BBB may be playing a role in preventing the disease in mice, where it may be compromised in humans suffering PCD. The development of a suitable animal model for PCD will greatly aid in identifying what events may be taking place to contribute to the neurological dysfunction and provide specific targets for treatment.

Another factor making it difficult to study the pathogenesis of PCD is the relatively short pathogenic process that PCD exhibits. Symptoms appear rapidly and then stabilize, so by the time a diagnosis is made the pathogenic process is likely over, making it difficult to study the progression of the disease directly in humans. PCD is also very rare so large scale studies are few and far between. Because of the rarity of PCD, most large scale studies group different types of PCD and compare the different types instead of comparing the same types to each other. It would be interesting to look at the differences that exist between patients who test positive for anti-Yo and do not develop PCD compared to those that do develop PCD. Differences in their serum tests and CSF content could provide clues to why some develop PCD and why others do not and provide insights into the pathogenesis behind PCD.

\section{CONCLUSION}

Anti-Yo positive paraneoplastic cerebellar degeneration is an immune-mediated disorder that occurs as a result of the presence of a malignant gynaecological or breast cancer tumour. Both anti-Yo and activated CTLs are products of the immune reaction against the underlying neoplasm and have been identified in PCD patients and have been implicated in playing a role in the aetiology of this disease. Studies thus far have been unable to show that either of these immune factors plays a direct role in Purkinje cell death. This is largely due to the lack of a suitable animal model for PCD, making it difficult to study the pathogenesis directly. Without a clear understanding of these mechanisms, it is difficult to implement effective treatment courses which thus far have been highly unsuccessful. The extensive loss of Purkinje cells that result from the immuno-pathogenesis of PCD causes severe irreversible neurological damage that greatly affects PCD patient's quality of life. It is important to understand PCD so it can be diagnosed quickly, not only to prevent further neurological damage but also so treatment of the underlying neoplasm can begin. Studying PCD requires a multidisciplinary approach which will not only provide insight into auto-immune neurological disorders but also provide a possible example of tumour immunity in humans which has implications in cancer research, and can provide a better understanding of the complex immune system and its interactions.

\section{REFERENCES}

1. Bataller L, Dalmau J. 2003. Paraneoplastic neurologic syndromes: approaches to diagnosis and treatment. Seminars in Neurology 23:215-224.

2. Denny-Brown D. 1948. Primary Sensory neuropathy with muscular changes associated with carcinoma. Journal of Neurology, Neurosurgery and Psychiatry 11:73-87.

3. Wilkinson PC, Zeromski J. 1965. Immunofluorescent detection of antibodies against neurones in sensory carcinomatous neuropathy. Brain: A Journal of Neurology 88:529-583.

4. Giometto B, Marchiori GC, Nicolao P, Scaravilli T, Lion A, Bardin PG, Tavolato B. 1997. Sub-acute cerebellar degeneration with anti-Yo autoantibodies: immunohistochemical analysis of the immune reaction in the central nervous system. Neuropathology and Applied Neurobiology 23:468-474. 
5. Sutton IJ, Steele J, Savage CO, Winer JB, Young LS. 2004. An interferon-gamma; ELISPOT and immunohistochemical investigation of cytotoxic $\mathrm{T}$ lymphocyte-mediated tumour immunity in patients with paraneoplastic cerebellar degeneration and anti-Yo antibodies. Journal of Neuroimmunology 150:98-106.

6. Brock S, Ellison D, Frankel J, Davis C, Illidge T. 2001. Anti-Yo antibody-positive cerebellar degeneration associated with endometrial carcinoma: case report and review of the literature. Clinical Oncology 13:476-479.

7. Cao Y, Abbas J, Wu X, Dooley J, Amburg Van AL. 1999. Anti-Yo positive paraneoplastic cerebellar degeneration associated with ovarian carcinoma: case report and review of the literature. Gynecologic Oncology 75:178-183.

8. Peterson K, Rosenblum MK, Kotanides H, Posner JB. 1992. Paraneoplastic cerebellar degeneration: A clinical analysis of 55 anti-Yo antibody-positive patients. Neurology 42:1931-1937.

9. Meglic B, Graus F, Grad A. 2001. Anti-Yo-associated paraneoplastic cerebellar degeneration in a man with gastric adenocarcinoma. Journal of the Neurological Sciences 185:135-138.

10. Fathallah-Shaykh H, Wolf S, Wong E, Posner J, Furneaux HM. 1991. Cloning of leucine-zipper protein recognized by the sera of patients with antibodyassociated paraneoplastic cerebellar degeneration. Proceedings of the National Academy of Sciences. $88: 3451-3454$.

11. Hida C, Tsukamoto T, Awano H, Yamamoto T. 1994. Ultrastructural localization of anti-Purkinje cell antibody-binding sites in paraneoplastic cerebellar degeneration. Archives of Neurology 51:555-558.

12. Graus F, Illa I, Agusti M, Ribalta T, Cruz-Sanchez F, Juarez C. 1991. Effect of intraventricular injection of anti-Purkinje cell antibody (anti-Yo) in a guinea pig model. Journal of the Neurological Sciences 106:82-87.

13. Furneaux HM, Rosenblum MK, Dalmau J, Wong E, Woodruff P, Graus F, Posner JB. 1990. Selective expression of Purkinje-cell antigens in tumour tissue from patients with paraneoplastic cerebellar degeneration. New England Journal of Medicine. 322:1844-1851.

14. Corradi JP, Yang C, Darnell JC, Dalmau J, Darnell RB. 1997. A post-transcriptional regulatory mechanism restricts expression of the paraneoplastic cerebellar degeneration antigen $\mathrm{Cdr} 2$ to immune privileged tissues. Journal of Neuroscience 17:1406-1415.

15. Sutton I, Winer JB. 2002. The immunopathogenesis of paraneoplastic neurological syndromes. Clinical Science 102:475-486.

16. Okano HJ, Park WY, Corradi JP, Darnell RB. 1999. The cytoplasmic Purkinje onconeural antigen Cdr2 downregulates c-Myc function: implications for neuronal and tumor cell survival. Genes Dev. 13:2087-2097.
17. Ruppert C, Goldowitz D, Wille W. 1986. Protooncogene c-myc is expressed in cerebellar neurons at different developmental stages. European Molecular Biology Organization Journal 5:1897-1901.

18. Sakai K, Kitagawa Y, Saiki S, Saiki M, Hirose G. 2004.Effect of a paraneoplastic cerebellar degenerationassociated neural protein on B-myb promoter activity. Neurobiology of Disease 15:529-533.

19. Sakai M, Sakai K, Saiki S, Kitagawa Y, Nakanishi M, Hirose G. 2005. Induction of humoral responses specific for paraneoplastic cerebellar degeneration-associated antigen by whole recombinant yeast immunization. Journal of Autoimmunity 24:203-208.

20. Albert ML, Darnell JC, Bender A, Francisco LM, Bhardwaj N, Darnell RB. 1998.Tumor-specific killer cells in paraneoplastic cerebellar degeneration. Nature Medicine 4:1321-1324.

21. Albert ML, Austin LM, Darnell RB. 2000. Detection and treatment of activated $\mathrm{T}$ cells in the cerebrospinal fluid of patients with paraneoplastic cerebellar degeneration. Annals of Neurology 47:9-17.

22. Westland KW, Pollard JD, Sander S, Bonner JG, Linington C, McLeod JG. 1999. Activated non-neural specific $\mathrm{T}$ cells open the blood-brain barrier to circulating antibodies. Brain: A Journal of Neurology 122:1283-1291.

23. Corriveau RA, Huh GS, Shatz, CJ. 1998. Regulation of class $1 \mathrm{MHC}$ gene expression in the developing and mature CNS by neural activity. Neuron 21:1-20.

24. Rojas I, Graus F, Keime-Guibert F, Reñé R, Delattre JY, Ramón JM, Dalmau J, Posner JB. 2000. Long-term clinical outcome of paraneoplastic cerebellar degeneration and anti-Yo antibodies. Neurology 55:713715 .

25. Storstein A, Knudsen A, Bjorge L, Meri S, Vedeler C. 2004. Heterogeneous expression of CD59 on human Purkinje cells. Neuroscience Letters 362:21-25.

26. Spiller OB, Criado-Garcia O, Rodriguez De Cordoba S, Morgan BP. 2000. Cytokine-mediated up-regulation of CD55 and CD59 protects human hepatoma cells from complement attack. Clin Exp Immunol 121:234-241.

27. Drlicek M, Bianchi G, Bogliun G, Casati B, Grisold W, Kolig C, Liszka-Setinek U, Marzorati L, Wondrusch E, Cavaletti G. 1997. Antibodies of the anti-Yo and anti-Ri type in the absence of paraneoplastic neurological syndromes: a long-term survey of ovarian cancer patients. Journal of Neurology 244:85-89.

28. Graus F, Delattre JY, Antoine JC, Dalmau J, Giometto B, Grisold W, Honnorat J, Smitt PS, Vedeler C, Verschuuren JJGM, Vincent A, Voltz R. 2004. Recommended diagnostic criteria for paraneoplastic neurological syndromes. Journal of Neurology, Neurosurgery, and Psychiatry 75:1135-1140.

29. Land R, Carter J, Houghton R, Atkinson K, Dalrymple C. 2005. Gynaecology meets neurology: paraneoplastic 
cerebellar degeneration. Australian and New Zealand Journal of Obstetrics and Gynecology 45:79-81.

30. Giannopoulou C. 2003. Navigating the paraneoplastic neurological syndromes. European Journal of Nuclear Medicine and Molecular Imaging 30:333-338.

31. Frings M, Antoch G, Knorn P, Freudenberg L, Bier U, Timmann D, Maschke M. 2005. Strategies in detection of the primary tumour in anti-Yo associated paraneoplastic cerebellar degeneration. Journal of Neurology 252:197-201.

32. Scheid R, Voltz R, Briest S, Kluge R, von Cramon DY. 2006. Clinical insights into paraneoplastic cerebellar degeneration. Journal of Neurology, Neurosurgery, and Psychiatry 77:529-530.

33. Widdess-Walsh P, Tavee JO, Schuele S, Stevens GH. 2003. Response to intravenous immunoglobulin in AntiYo associated paraneoplastic cerebellar degeneration: case report and review of the literature. Journal of Neuro-Oncology 63:187-190.

34. Shams'ili S, Grefkens J, de Leeuw B, van den Bent M, Hooijkaas H, van der Holt B, Vecht C, Sillevis Smitt P. 2003. Paraneoplastic cerebellar degeneration associated with antineuronal antibodies: analysis of 50 patients. Brain: A Journal of Neurology 126:1409-1418.

35. Candler PM, Hart PE, Barnett M, Weil R, Rees JH. 2004. A follow up study of patients with paraneoplastic neurological disease in the United Kingdom. Journal of Neurology, Neurosurgery and Psychiatry 75:1411-1415.

36. Sakai K, Shirakawa T, Kitagawa Y, Li Y, Hirose G. 2001. Induction of cytotoxic T lymphocytes specific for paraneoplastic cerebellar degeneration-associated antigen in vivo by DNA immunization. Journal of Autoimmunity 17:297-302. 